\title{
Necropsy diagnosis of myocarditis: a retrospective study using CD45RO immunohistochemistry
}

\author{
K M Feeley, J Harris, S K Suvarna
}

\begin{abstract}
Aim-To use CD45RO immunohistochemistry to investigate the numbers of $T$ lymphocytes found in sections of myocardium from a routine necropsy series, and to determine the incidence of myocarditis in this series.

Methods-Myocardial sections from 163 routine hospital necropsies were stained with CD45RO and the numbers of positive lymphocytes $/ \mathrm{mm}^{2}$ were counted. The results were correlated with the $H / E$ opinion and the clinical context of the necropsy. Results-Most (143) cases showed low numbers (0-3) of CD45RO positive lymphocytes $/ \mathrm{mm}^{2}$. Fifteen cases showed 7-13 positive lymphocytes $/ \mathrm{mm}^{2}$, comprising a wide variety of clinical conditions, generally with no specific cardiac pathology. Five cases showed 14 or more positive lymphocytes $/ \mathrm{mm}^{2}$, comprising one case of active myocarditis, three cases of cardiac transplant rejection, and one posttransplant lymphoproliferative disorder, all conditions in which large numbers of lymphocytes would be expected.

Conclusions-The incidence of myocarditis in our series was $0.6 \%$. In most cases the normal myocardium has a low $\mathrm{T}$ lymphocyte count $\left(0-3 / \mathrm{mm}^{2}\right)$. In some cases immunohistochemistry shows more positive cells than would have been expected on light microscopy. Immunohistochemistry is a useful and reliable means of confirming a diagnosis of myocarditis. The results support the conclusion of the 1997 ISFC task force that 14 or more lymphocytes or macrophages $/ \mathrm{mm}^{2}$ of myocardium in the appropriate clinical context is a reliable threshold for the diagnosis of chronic myocarditis.

(F Clin Pathol 2000;53:147-149)
\end{abstract}

Keywords: myocarditis; immunohistochemistry; $\mathrm{T}$ lymphocytes; necropsy

Department of Histopathology, Northern General Hospital, Herries

Road, Sheffield S5

7AU, UK

K M Feeley

J Harris

S K Suvarna

Correspondence to: Dr Suvarna email: s.k.suvarna@ sheffield.ac.uk cases of heart failure. ${ }^{2}$ Since the publication of the Dallas criteria, ${ }^{3}$ the histological assessment of the myocardium on endomyocardial biopsy has become the gold standard for the diagnosis of myocarditis. In 1997 the International Society and Federation of Cardiology (ISFC) revisited the Dallas criteria, and one of their recommendations was that 14 or more lymphocytes and/or macrophages per $\mathrm{mm}^{2}$ of myocardium was a suitable threshold for the diagnosis of chronic myocarditis.

In the present study we have used CD45RO immunohistochemistry to examine the numbers of $\mathrm{T}$ lymphocytes found in sections of myocardium from an unselected hospital necropsy series. We aimed to determine the incidence of myocarditis in the series, to investigate the numbers of $\mathrm{T}$ lymphocytes found in the "normal" myocardium, and to assess the value of immunohistochemistry in the evaluation of a diagnosis of myocarditis. Our particular interest was to test the validity of the conclusion of the ISFC task force that 14 or more lymphocytes and/or macrophages per $\mathrm{mm}^{2}$ of myocardium is suggestive of chronic myocarditis.

\section{Methods}

Paraffin embedded myocardial tissue blocks from 163 necropsy cases performed by the same pathologist over a five year period at our centre were retrieved. One block per case was used, as this is what was taken as routine at our hospital necropsies where no specific myocardial pathology was suspected. Haematoxylin and eosin (H\&E) sections were prepared and $5 \mu \mathrm{m}$ sections were also stained with CD45RO (Dako, OPD4) in accordance with the manufacturer's instructions. At least 20 high power fields of each section were examined on light microscopy in a stepwise fashion using a "one field down, two fields over" methodology, and the number of CD45RO positive lymphocytes/ $\mathrm{mm}^{2}$ was counted. It was noted that some macrophages and polymorph neutrophils were also highlighted by the immunohistochemistry. However, in most cases the cell type could be discerned morphologically. Where distinction was not possible the cell was included in the count. Specific care was taken not to count any cell within a blood vessel or capillary. The immunohistochemical and $\mathrm{H} \& \mathrm{E}$ sections were assessed independently of each other by two pathologists. The cell count $/ \mathrm{mm}^{2}$ was established and the results were checked against the necropsy diagnosis and $\mathrm{H} \& \mathrm{E}$ opinion.

\section{Results}

The cases had an age range of 14 to 94 years; 55 were female and 108 male. The results of the cell count showed the majority (143 cases) to have less than seven positive lymphocytes/ 
Table 1 Breakdown of myocardial CD45RO lymphocyte counts by case frequency

\begin{tabular}{ll}
\hline Cells $/ \mathrm{mm}^{2}$ & $n$ \\
\hline 0 & 33 \\
1 & 39 \\
2 & 38 \\
3 & 12 \\
4 & 10 \\
5 & 9 \\
6 & 2 \\
$7 / 7+$ & 20 \\
\hline
\end{tabular}

Table 2 Analysis of disorder seen versus CD45RO cell count

\begin{tabular}{ll}
\hline Disorder & Cell count \\
\hline Myocarditis & 97 \\
Cardiac rejection & $10,53,20,196$ \\
PTLPD & 8,38 \\
Pancreatitis & 8 \\
Pulmonary embolism & 8 \\
Ischaemic heart disease & $9,10,9,7$ \\
Ulcer & 7 \\
Pneumocystis carinii/AIDS & 9 \\
Neoplasia* & $11,8,8,11,9$ \\
\hline
\end{tabular}

^Lung, pancreas, gall bladder, rectum, mesothelioma. PTLPD, post-transplant lymphoproliferative disorder.

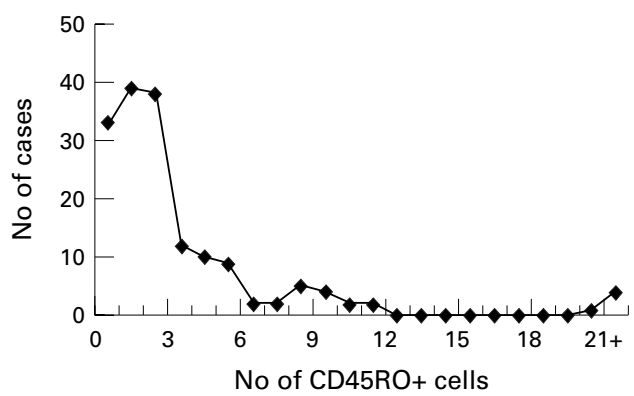

Figure 1 Graphical representation of myocardial $\mathrm{CD} 45 \mathrm{RO}+$ cell density $/ \mathrm{mm}^{2}$ by case frequency.

$\mathrm{mm}^{2}$ (table 1). It was noted that most had very few positive cells or none at all. However, 20 cases in which seven or more CD45RO positive lymphocytes were present were identified. From these 20 cases, two major groups emerged. The first group showed 7-13 CD45RO positive lymphocytes $/ \mathrm{mm}^{2}$, and included malignant neoplasms, treated cardiac transplant rejection, post-transplant lymphoproliferative disorder, pancreatitis, ischaemic heart disease (not showing acute myocardial infarction), pulmonary embolism, peptic ulcer, and pneumonia. The second group showed 14 or more CD $45 \mathrm{RO}$ positive lymphocytes $/ \mathrm{mm}^{2}$, and comprised three cases of cardiac transplant rejection, one post-transplant lymphoproliferative disorder, and one case of active myocarditis. With both of these groups it was noted that there was a substantial field to field variation in the number of positive cells in a section, ranging from 0 to $>15$ positive cells per high power field, and that positive cells tended to occur in aggregates in a patchy manner. However, we found that examination of 20 random high power fields gave an average per $\mathrm{mm}^{2}$ that was not altered by examining further fields. The two groups are displayed in table 2 , and a graphical representation of all the results is given in fig 1.

\section{Discussion}

Prompt and accurate diagnosis of myocarditis is important given the possibilities of early cardiac death from fulminant cardiac failure or slow progression to the state of dilated cardiomyopathy, which accounts for $25 \%$ of cases of heart failure and which in a proportion of cases is thought to be caused by chronic myocarditis. ${ }^{2}$ It also has relevance when one is considering possible cardiac transplantation. The precise incidence of myocarditis at necropsy has varied considerably in published reports, but since the introduction of the endomyocardial biopsy the accurate diagnosis of myocarditis has become a necessity. Although numerous grading schemes were employed initially, the publication of the Dallas criteria $^{3}$ has permitted standardisation worldwide.

The Dallas criteria were revolutionary, although they allowed for "grey" areas such as borderline myocarditis and difficulties in interpreting resolving myocarditis. Recent advances in immunohistochemistry have led to the understanding that a variety of cells, including macrophages, $\mathrm{T}, \mathrm{B}$, and NK (natural killer) lymphocytes, all play a role in the acute phase of myocyte damage. ${ }^{4-6}$ The chronic/persistent phase, in contrast, is characterised by an inflammatory infiltrate in the myocardium consisting almost exclusively of $\mathrm{T}$ lymphocytes and macrophages. ${ }^{7}$ Several studies have shown that various cytokines and nitric oxide also play a role in the chronic phase of myocyte damage. $^{8-10}$ The pathogenesis of the chronic inflammatory response is still largely unclear, however, but some investigators have proposed that it may represent a form of autoimmune cytotoxic $\mathrm{T}$ cell response. ${ }^{11}{ }^{12} \mathrm{With}$ regard to the numbers of myocardial inflammatory cells typically found in chronic myocarditis, Kuhl et al examined a cohort of patients with dilated cardiomyopathy, and concluded that the finding of seven or more T lymphocytes $/ \mathrm{mm}^{2}$ was abnormal and suggestive of chronic myocarditis. ${ }^{13}$ The 1997 ISFC task force reconsidered the Dallas criteria, and one of their conclusions was that 14 or more lymphocytes and/or macrophages per $\mathrm{mm}^{2}$ was a reliable threshold for the diagnosis of chronic myocarditis. ${ }^{14}$ This was the subject of further discussion at the European School of Cardiovascular Pathology in Amsterdam, October 1998. The normal myocardium contains 1.54.0 T lymphocytes $/ \mathrm{mm}^{2}{ }^{15}$

However, most of the studies to date looking at myocarditis have concentrated on stored tissue samples from positive myocarditis cases and have used controls largely derived from non-transplant cardiac surgery. In short, assessment of a normal population has not been undertaken. Furthermore, as there appeared to be some debate as to the incidence of myocarditis at necropsy, with figures ranging from 1.06 to $10 \%,{ }^{16-20}$ we chose to assess myocarditis retrospectively within an unselected necropsy population.

A wide range of immunohistochemical reagents staining for $\mathrm{T}$ lymphocytes and macrophages is available. We chose CD45RO 
as we were conversant with it in our daily practice. We were aware that this antibody cross reacts with polymorph neutrophils and some activated macrophages. However, we found that neutrophils could easily be distinguished morphologically, and in most cases macrophages could also be discerned. Where there was doubt about the nature of a positively staining cell, it was included in the count. This was not a problem as the 1997 ISFC group recognised that the inflammatory infitrate in chronic myocarditis comprises both macrophages and $\mathrm{T}$ lymphocytes. We took specific care to exclude positively staining cells present in capillaries or larger vessels. We also excluded all cases of acute myocardial infarction.

Our results indicate that in most cases myocardial tissue sampled at routine necropsy has a low lymphocyte count ( $0-3$ cells) per $\mathrm{mm}^{2}$. This supports the previous reports on the subject. Those cases in our study showing 14 or more positive lymphocytes and/or macrophages per $\mathrm{mm}^{2}$ of myocardium comprised one case of active myocarditis, three of cardiac transplant rejection, and one post-transplant lymphoproliferative disorder. These are conditions quite remote from chronic myocarditis and in which large numbers of myocardial lymphocytes and macrophages would be expected. In contrast, those cases showing 7-13 lymphocytes $/ \mathrm{mm}^{2}$ covered a wide range of different pathological entities, including a variety of non-cardiac conditions such as pulmonary embolism, peptic ulcer, pneumonia, and pancreatitis, where a myocardial inflammatory infiltrate would not be expected, together with one case of cardiac transplant rejection undergoing treatment, and one case of posttransplant lymphoproliferative disorder. These findings indicate that up to 13 lymphocytes and/or macrophages per $\mathrm{mm}^{2}$ of myocardium may be found in conditions involving no specific cardiac pathology. Our work therefore supports the recommendation of the ISFC task force that 14 or more lymphocytes and/or macrophages per $\mathrm{mm}^{2}$ of myocardium in the appropriate clinical context is a reliable threshold for the diagnosis of chronic myocarditis.

We noted in addition that the immunohistochemistry produced higher scores than we had expected from light microscopy alone. This underlines the value of immunohistochemistry in the diagnosis of myocarditis. In so doing, it poses the question as to the role of myocardial lymphocytes in non-myocarditis cases: have they any specific and significant cellular interaction, or are they just "passing through"?

We are reassured to find that our results regarding the incidence of myocarditis $(0.6 \%)$ parallels larger series looking at necropsies. We therefore also recommend the use of immunohistochemistry in the necropsy diagnosis of myocarditis, particularly if standard histology fails to provide a definite answer. We recommend the examination of at least 20 high power fields from at least one block. This has particular relevance in cases of sudden cardiac death and in patients who have suffered progressive cardiac failure.
Given the range of immunohistochemical reagents that can reliably identify $\mathrm{CD} 3$ and CD68, it might be suggested that these two antibodies should be used in preference to CD45RO. Indeed, as a result of this study, we have chosen to use CD3 and CD68 in future work. However, we recognise the difference between the teaching/research laboratory and general laboratory, and also the impact of cost in terms of reagents and time, and we continue to feel that CD45RO would suffice for the purposes we have outlined.

In conclusion, we feel that this study has shown the value of immunohistochemistry in the diagnosis of myocarditis, and has validated the recommendations of the ISFC task force. We have also provided evidence to support previously published data showing the low incidence of myocarditis found at necropsy in the general population. The choice of antibodies used appears to be an individual decision. Further investigations examining the role of lymphocytes in the myocardium outside myocarditis are indicated by this study.

1 Aretz HT, Billingham ME, Edwards WD, et al. Myocarditis: a histopathologic definition and classification. $A m$ f Cardiovasc Pathol 1987;1:3-14.

2 Sole MJ, Liu P. Viral myocarditis: a paradigm for understanding the pathogenesis and treatment of dilated cardiomyopathy. F Am Coll Cardiol. 1993;22(suppl A):99$105 \mathrm{~A}$

3 Aretz H. Myocarditis: the Dallas criteria. Hum Pathol 1987; 18:619-24.

4 Kuhl U, Noutsias M, Schultheiss HP. Immunohistochemistry in dilated cardiomyopathy. Eur Heart $\mathcal{F}$ 1995;16(suppl O): $100-6$.

5 Yazaki Y, Isobe M, Yamazaki S, et al. Ultrastructural and immunohistochemical analysis of biopsy-proven chronic active myocarditis with numerous clusters of lymphocytes. Virchows Arch 1998;433:161-6.

6 Henke A, Huber S, Stelzner A, et al. The role of CD8+ T-lymphocytes in coxsackie B3-induced myocarditis. $\mathcal{f}$ Virol 1995;69:6720-8.

7 Schwimmbeck PL, Badorff C, Rohn C, et al. The role of sensitized T-cells in myocarditis and dilated cardiomyopathy. Int $\mathcal{F}$ Cardiol 1996;54:117-25.

8 Bowles NE, Towbin JA. Molecular aspects of myocarditis. Curr Opin Cardiol 1998;13:179-84.

9 Matsumori A. Molecular and immune mechanisms in the pathogenesis of cardiomyopathy-role of viruses, cytokines, and nitric oxide. fpn Circ f 1997;61:275-91.

10 Matsumori A. Cytokines in myocarditis and cardiomyopathies. Curr Opin Cardiol 1996;11:302-9.

11 Caforio AL, Goldman JH, Haven AJ, et al. Evidence for autoimmunity to myosin and other heart-specific autoantigens in patients with dilated cardiomyopathy and their gens in patients with dilated cardiom
relatives. Int $\mathcal{F}$ Cardiol 1996;54:157-63.

12 Malkiel S, Kuan AP, Diamond B. Autoimmunity in heart disease: mechanisms and genetic susceptibility. Mol Med Today 1996;2:336-42.

13 Kuhl U, Noutsias M, Seeberg B, et al. Immunohistological evidence for a chronic intramyocardial inflammatory process in dilated cardiomyopathy. Heart 1996;75:295-300.

14 Maisch B. Classification of cardiomyopathies according to the WHO/ISFC Task Force-more questions than answers? Med Klin 1998;93:199-209.

15 Mues B, Brisse B, Zwadlo G, et al. Phenotyping of macrophages with monoclonal antibodies in endomyocardial biopsies as a new approach to diagnosis of myocarditis. Eur Heart f 1990;11:619-27.

16 Gravanis MB, Sternby NH. Incidence of myocarditis. A 10-year autopsy study from Malmo, Sweden. Arch Pathol Lab Med 1991;115:390-2.

17 Gore I, Saphir O. Myocarditis: a classification of 1402 cases. Am Heart F 1947;34:827-30.

18 Kline JK, Kline TS, Saphir O. Myocarditis in senescence. Am Heart F 1963;65:446-57.

19 Gore I, Kline JK. Pericarditis and myocarditis. In: Gould SE. ed. Pathology of the heart and blood vessels, 3rd ed. Springfield, Ill: Charles C Thomas, 1968:731-59.

20 Abelmann WH. Myocarditis. N Engl f Med 1966;275:8324. 